\title{
Green Chemistry Formation of Stable Ag Nanoparticles (AgNPs) In Isopropanol Solvent
}

\author{
SYUKRI ARIEF*1, PUTRI HIDAYANI', LUSI AFERTA ${ }^{1}$, ZULHADJRI', \\ TAKAYUKI BAN ${ }^{2}$ and YUTAKA OHYA ${ }^{2}$
}

\author{
1Department of Chemistry, Faculty of Mathematic and Sciences, \\ Andalas University, Kampus Limau Manis, Padang - Indonesia. \\ ${ }^{2}$ Department of Chemistry and Biomelecular Science, \\ Faculty of Engineering, Gifu University, Yanagido 1 - 1, Gifu - Japan. \\ ${ }^{\star}$ Corresponding author E-mail: syukriarief@gmail.com
}

http://dx.doi.org/10.13005/ojc/330109

(Received: October 31, 2016; Accepted: January 04, 2017)

\begin{abstract}
Several bioreduction processes become appreciation for the synthesis of silver nanoparticles (AgNPs) because of their multiple applications, cost effective and eco-friendly. The main problems in the synthesis of AgNPs are colloidal systems agglomeration and damage due to precipitation. In this study, it has been investigated the use of Uncaria Gambir Roxb leaf extract as bioreductor and capping agent on the synthesis of AgNPs and isopropanol as a solvent. XRD studies showed that the AgNPs are crystalline fcc (face center cubic) without another phase and AgNPs size in isopropanol solution was smaller than aqueous solution. TEM images indicated that the obtained AgNPs are predominantly spherical shape, with sizes in the range of 10 to $30 \mathrm{~nm}$.
\end{abstract}

Keywords: Silver nanoparticles, bioreductor, isopropanol solvent, Uncaria Gambir Roxb.

\section{INTRODUCTION}

In recent years, research on the synthesis of metal nanoparticles has been widely discussed and has become a major topic due to their new or improved chemical, optical, electric, and magnetic properties compared to their bulks ${ }^{1}$. Among them, silver nanoparticles (AgNPs) have been a focus of many researchers not only because of their properties but also because silver nanoparticles have been widely used as an antimicrobial property ${ }^{2}$. Their properties strongly depend on their size and shape, and therefore it is very important to be able to finely control the morphology of the nanoparticles. 
Several methods have been used in the formation of AgNPs such as the reduction in solution, electroless, photochemical reduction, hydrothermal, and assisted-microwave synthesis. So far, several weaknesses have been found, including the need for sophisticated and more expensive equipment. They also have high toxicity properties and more particularly difficult to obtain a stable colloid.

A green synthesis method is an attractive option at this time where the synthesis of AgNPs uses various extracts of the leaves of plants including the most leaves that have flavonoid compounds in the presence of polyphenols structure such as Rumex hymenasepalus ${ }^{3}$, Artocarpus heterophyllus ${ }^{4}$, Camellia Sinensis 5 , Mollugo nudicaulis ${ }^{6}$ and Azadirachta indica ${ }^{7}$ which can reduce $\mathrm{Ag}(\mathrm{I})$ to $\mathrm{Ag}(0)$. Ramachandran et $\mathrm{al}^{8}$ and Siavash Iravani ${ }^{9}$ have reviewed various kinds of plant extracts that have been used in the synthesis of metal nanoparticles, especially Ag nanoparticles. In general, the resulting nanoparticles of this process have size in the range of nanosize with spherical and triangular structures ${ }^{10-12}$.
In the case of metal oxides, solvent is a factor that determines the stability and also controls the size and shape of the particles ${ }^{13}$. In our previous studies, it has been reported that the use of leaf extracts bioreducing gambier was able to act as reducing agent in the formation of silver nanoparticles in aqueous solvent. AgNPs with sizes of $25-40 \mathrm{~nm}$ have been obtained with the help of a hydrothermal process ${ }^{14}$. In this study we report biosynthesis of AgNPs by reduction of silver nitrate $\left(\mathrm{AgNO}_{3}\right)$ in isopropanol solution using isopropanol - Gambier leaf extract in order to control the shape and size of silver nanoparticles.

\section{MATERIALS AND METHODS}

\section{Plant material and preparation of extracts}

All chemicals used for this reaction were of analytical grade and obtained from Merck without any further purifications. Gambir leaves were collected from agricultural field located at Payakumbuh West Sumatera, Indonesia. Fresh leafs of Gambir were washed thoroughly with double distilled water. Dried leaf were incised and crushed into small pieces. Approximately $5 \mathrm{~g}$ of those finely Gambir

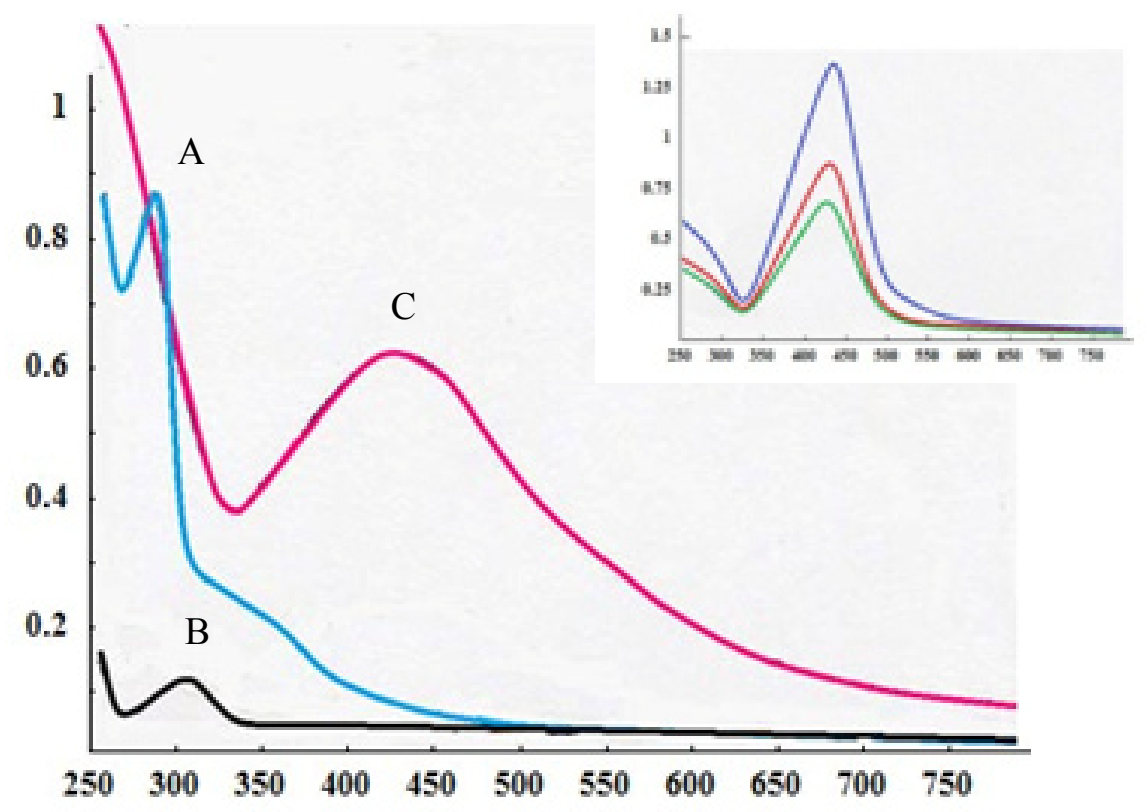

Fig. 1: The absorption of the plant extract (A), pure $\mathrm{AgNO}_{3}$ solution (B), and a sample of AgNPs with reaction time of $4 \mathrm{~h}(\mathrm{C})$ 
were weighted and transferred into a $200 \mathrm{ml}$ beaker containing $50 \mathrm{ml}$ isopropanol, mixed well and boiled for 30 minutes. The extract obtained was filtered

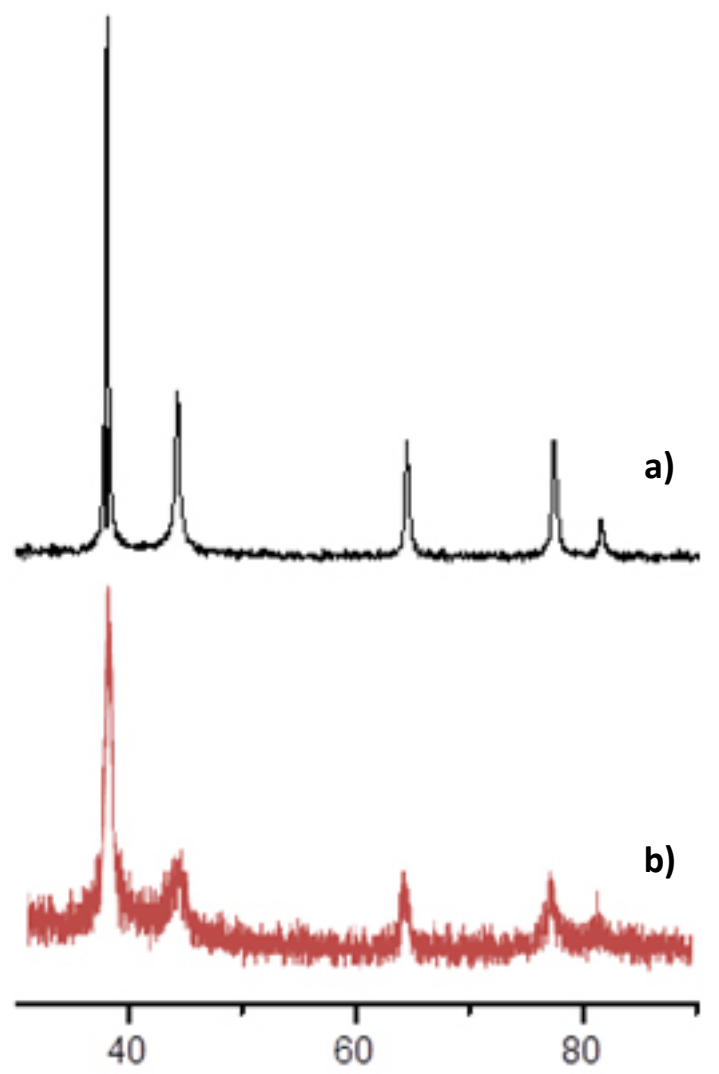

Fig. 2: XRD patterns of synthesized AgNPs using different solvent, a) in aqueous solution, b) Isopropanol solution

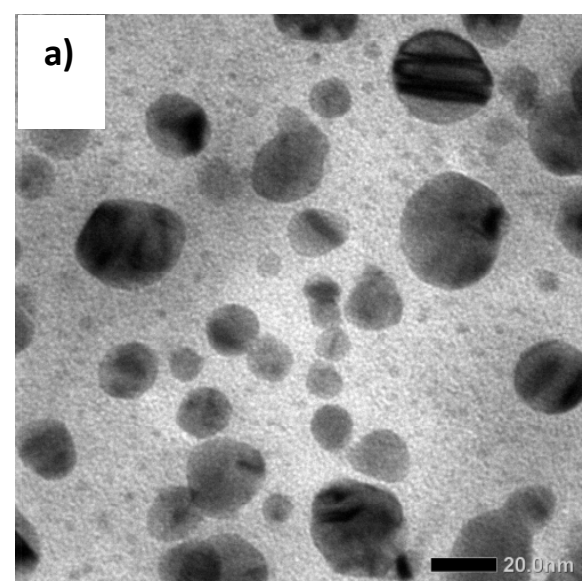

through filter paper and the filtrate was diluted and collected to $250 \mathrm{ml}$ with isopropanol. The solution was kept in refrigerator for further use.

\section{Synthesis of AgNPs}

The Ag Nanoparticles from Gambier leaves extract was prepared as previously described method with slightly modifications ${ }^{14}$. Isopropanol solution (5 $\mathrm{mM}$ ) of silver nitrate $\left(\mathrm{AgNO}_{3}\right)$ was prepared and used for the synthesis of silver nanoparticles. An amount of $10 \mathrm{ml}$ of extract was added to $40 \mathrm{ml}$ of 5 $\mathrm{mM} \mathrm{AgNO}_{3}$ solution. After addition of extracts, the color of the $\mathrm{AgNO}_{3}$ solution altered to brown. The effect of reaction times on the synthesis of silver nanoparticles was carried out and evaluated every 10 minutes. The precipitate was separated by high speed centrifugation at $6000 \mathrm{rpm}$. The separated solid mass was washed three times with ethanol to remove the organic alcohol soluble impurities. Product was dried in oven at 50C for 2-3 h. Finally, it was transferred into light resistant containers for further characterization.

\section{Characterization}

UV-vis spectroscopic studies were carried out on a Thermo spectrophotometer. The sample for XRD measurement was prepared by depositing a dried sample on a slide and the diffraction pattern was recorded on a PANalytic X'PERT-PRO $X$-ray spectrometer. The size of the nanoparticles was calculated by the Scherer's equation. High resolution-transmission electron microscopic (HR-

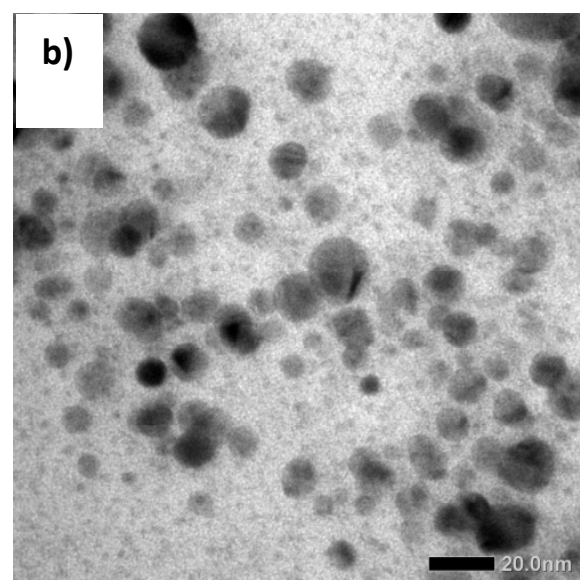

Fig. 3: TEM images of the AgNPs at different solvents, a) in aqueous solution, b) Isopropanol solution 
TEM) measurements were done using a Shimadzu CTLA-4 microscope.

\section{RESULTS AND DISCUSSIONS}

\section{UV-vis analysis}

The addition of the extract into $5 \mathrm{mM}$ of $\mathrm{AgNO}_{3}$ solution changed color from pale yellow to brownish within 5 minutes. The color is more intense with increasing time of reaction. The change in color is due to the strong absorption of visible light due to excitation of the nanoparticle surface plasmons ${ }^{15}$. This is a strong indication of the formation of silver nanoparticles. In Figure 1, the curves demonstrate the absorption of the the plant extract $(A)$, pure $\mathrm{AgNO}_{3}$ solution (B), and a sample of AgNPs with reaction time for $4 \mathrm{~h}(\mathrm{C})$. The polyphenol peak (observed in the Gambir extract) is also clearly visible around $278 \mathrm{~nm}$, while the UV-Vis spectra of the $\mathrm{AgNO}_{3}$ solution has a peak around $305 \mathrm{~nm}$, as expected for Ag+ ions. These spectral studies identify the formation of stable nanoparticles of silver (insert: higest peak for one week). The maximum absorption shows the appearance of a single and strong surface plasmon resonance band absorption peak centered at about $427 \mathrm{~nm}$, which indicates that these particles are isotropic in shape and relatively uniform in size.

\section{X-Ray diffraction (XRD)}

The XRD analysis is performed to confirm the crystalline nature of AgNPs that prepared by using Gambier extract as bioreductor. Various Bragg's reflections are clearly visible in silver XRD pattern, which are corresponding to the (111), (200), (220) and (311) set of lattice planes, and also agreed by joint committee of powder diffraction standard (JCPDS) Card No- 04-0783 (Fig. 2). On the other hand, the difference in solvent gives different peak sharpness and heights. Peaks obtained from aqueous solution are sharper and higher than isopropanol solution. Based on the equation Scherer, a sharp and height peak gives larger crystal size than the width and low peak. This will be proved later by TEM.

\section{Transmission Electron Microscope (TEM)}

The typical TEM images of AgNP-Gambier Isopropanol and aqueous solution are given in Fig. 3.The images show that the nanoparticles synthesized using Isopropanol as solvent are almost spherical and slightly polydispersed. The size of AgNPs is ca. $10-25 \mathrm{~nm}$ according to the TEM image, and that is consistent with the published data for the synthesis of AgNPs by the chemical reduction method ${ }^{16}$. The TEM images show good stabilization of silver nanoparticles due to interaction with the Gambier - isopropanol solution. Based on ionic strength, water media is more powerful than isopropanol media. Their electrophoretic mobility is freer in aqueous media due to faster growth of nanoparticles. Organic media (isopropanol solvent) is absorbed on to these nanoparticles significantly reducing the aggregation and improving its AgNPs stability.

\section{CONCLUSION}

We have demonstrated a green chemistry approach for the synthesis of silver nanoparticles at ambient reaction conditions using Uncaria Gambier Roxb leaf extract and isopropanol solvent. The leaf extract acts as reducing agent as well as stabilizing/ capping agent. These biosynthesized AgNPs are highly stable for several weeks in isopropanol solution. XRD studies show that the AgNPs are crystalline fcc (face center cubic) and particles size is of $20 \mathrm{~nm}$ using isopropanol solution. TEM images indicate that the obtained AgNPs are predominantly spherical shape, with sizes in the range of 10 to 30 $\mathrm{nm}$.

\section{ACKNOWLEDGEMENTS}

The authors would like to thank the Higher Education Ministry of Indonesia via Andalas University that has funded this research through Grants of Cooperation Foreign Affairs and International Publications with contract No. 020/SP2H/LT/DRPM/ II/2016. 


\section{REFERENCES}

1. Schmid, G.(ed.), Nanoparticles : From Theory to Application, 2nd Ed., Wiley-VCH, Singapore, 2010

2. David, P. Silver Nanoparticles, Published by In-The, India, 2010

3. Leon, E. R.; Palomares, R. I.; Navarro, R. E.; Urbina, R. H.; Tanori, J.; Palomares, C. I.; Maldonado, A. Nanoscale Res.Let. 2013, 8, 318 -324

4. Thirumurugan, A.; Tomy, N.A.; Ganesh, R.J.; Gobikrishnan, S. Der Pharma Chemica, 2010, 2(6), 279-284

5. Loo, Y.Y.; Chieng, B.W.; Nishibuchi, M.; Radu, S. Int. J. Nanomedicine, 2012, 7, 4263-4267

6. Anarkali, J.R.; Vijayanti, D.; Rajathi, K.; Sridhar, S. Indo American J. of Pharm. Res, 2012, 4, 1436 - 1442.

7. Shankar, S.S.; Rai, A.; Ahmad, A.; dan Sastry, M. J. Colloid Interface Sci., 2004, 275(4), 496502.
8. Ramachandran R.; Krishnaraj C.; Stacey L. H.; Soon-IIY.; Thangavel K. Ind. Crops Prod., 2015, 70, 356-373

9. Siavash I., Green Chem., 2011, 13, 26382650

10. Singh C.; Baboota, R. K.; Naik, P. K.; Singh H. Adv. Mater. Lett. 2012, 3(4): 279-285.

11. Leela A. and Vivekanandan M. African J.of Biotech., 2008, 7(17): 3162-3165.

12. Zargar M.; Hamid, A. A.; Bakar, F. A.; Shamsudin, M. N.; Shameli K.; Jahanshiri F.; Farahani F. Molecules , 2011, 16: 66676676

13. Kraynov A. and Thomas E.M. Application of Ionic liquid in Science and Technology, Edited by Scott T. H., In-tech, Croatia, 2011

14. Syukri A.; Vivi G.; Diana V.W.; Zulhadjri Z.; Ban T. and Ohy Y. J. Chem. Pharm. Res. 2015, 7(9S), 189-192

15. Ray PC, Chem Rev., 2010, 110, 53325365 